\title{
Magnetic fields of AGNs and standard accretion disk model: testing by optical polarimetry
}

\author{
N. A. Silant'ev ${ }^{1}$, M. Yu. Piotrovich ${ }^{2}$, Yu. N. Gnedin ${ }^{2}$, and T. M. Natsvlishvili ${ }^{2}$ \\ ${ }^{1}$ Instituto Nacional de Astrofísica, Óptica y Electrónica, Luis Enrique Erro 1, Apartado Postal 51 y 216, 72840, Tonantzintla, \\ Puebla, México \\ e-mail: silant@inaoep.mx \\ 2 Central Astronomical Observatory at Pulkovo of Russian Academy of Sciences, 196140, Saint-Petersburg, \\ Pulkovskoe shosse 65, Russia
}

Received 1 September 2008 / Accepted 15 July 2009

ABSTRACT

\begin{abstract}
We have developed the method that allows us to estimate the magnetic field strength at the horizon of a supermassive black hole $(\mathrm{SMBH})$ through the observed polarization of optical emission of the accreting disk surrounding SMBH. The known asymptotic formulae for the Stokes parameters of outgoing radiation are azimuthal averaged, which corresponds to an observation of the disk as a whole. We consider two models of the embedding 3D-magnetic field, the regular field, and the regular field with an additional chaotic (turbulent) component. It is shown that the second model is preferable for estimating the magnetic field in NGC 4258. For estimations we used the standard accretion disk model assuming that the same power-law dependence of the magnetic field follows from the range of the optical emission down to the horizon. The observed optical polarization from NGC 4258 allowed us to find the values $10^{3}-10^{4}$ Gauss at the horizon, depending on the particular choice of the model parameters. We also discuss the wavelength dependencies of the light polarization, and possibly applying them for a more realistic choice of accretion disk parameters.
\end{abstract}

Key words. black hole physics - magnetic fields - accretion, accretion disks - polarization

\section{Introduction}

It is now commonly accepted that active galactic nuclei (AGNs) and quasars (QSOs) frequently possess the magnetized accretion disks (see, for example, the reviews of Blaes 2003; Moran 2008 on NGC 4258). There are many models of the accretion disk structures (see Pariew et al. 2003, and references therein). The best known and most frequently used is the standard model of Shakura \& Sunyaev (1973). The polarimetric observations frequently demonstrate that AGNs and QSOs have polarized emission in different wavelength ranges, from ultraviolet to radio waves, in continuum and in the line emission (see Martin et al. 1983; Webb et al. 1993; Impey et al. 1995; Wilkes et al. 1995; Barth et al. 1999; Smith et al. 2002; Modjaz et al. 2005). These papers discuss the different mechanisms for the origin of the observed polarization: the light scattering in accretion disks, which happens on both free and bound electrons, synchrotron radiation of charged particles. These mechanisms can work in different structures such as the plane and warped accretion disks and toroidal clumpy rings, surrounding the accretion disks and jets. Frequently different models are proposed to explain the same source. There are a lot of papers devoted to different aspects of the structure and emission of AGNs and QSOs. Many theoretical papers propose the possible behavior of a magnetic field in these objects.

In this paper we develop the technique of estimating the magnetic fields in different parts of plasma accretion disks. Especially interesting is the estimation of magnetic field in the horizon of the supermassive black holes in AGNs. The main idea is to use the observed integral polarization from magnetized plasma accretion disks. We use the known fact that the Faraday rotation of polarization plane changes both the values of integral polarization degree $p$ and position angle $\chi$. The observed spectra $p(\lambda)$ and $\chi(\lambda)$ acquire very specific forms due to Faraday rotation. The detailed discussion and calculations of these effects are presented in Silant'ev (1994), Dolginov et al. (1995), Gnedin \& Silant'ev (1997), Agol \& Blaes (1996), etc. The observed polarization possesses the information about the magnetic field in magnetized electron atmospheres and can serve for estimating the field. In Gnedin et al. (2006) the method was considered for pure vertical magnetic field $\boldsymbol{B}_{\|}$and without the correct azimuthal averaging of the asymptotic formulae (4).

For estimating the magnetic field we use the simple approximate formulae (Silant'ev 2002) that represent solutions to a number of "standard" problems of the radiative transfer theory in magnetized electron atmospheres, namely, the Milne problem and the cases when the sources of thermal radiation are distributed homogeneously, linearly, and exponentially in an optically thick atmosphere. These "standard" solutions allow us to approximate the solution of problem with a more complex distribution of thermal sources inside the atmosphere, because the latter can be presented as a superposition of "standard" sources.

For the optically thick accretion disks, the solution of the Milne problem is used, i.e. the case where the sources of thermal radiation are located far from the surface. The polarization and angular intensity distribution of outgoing radiation for non-magnetized electron atmosphere is presented in the known Chandrasekhar's book (see Chandrasekhar 1950). The numerical solution to Milne's problem for a magnetized electron atmosphere with the magnetic field $\boldsymbol{B}_{\|}$parallel to the normal $\boldsymbol{N}$ to an atmosphere is presented in Agol \& Blaes (1996), and Shternin et al. (2003). The numerical solution to this problem for the 
turbulent magnetized atmosphere is given in Silant'ev (2007). In this paper the approximate formulae for Milne's problem are also generalized for the case of a turbulent atmosphere. It is very important that the approximate formulae of Silant'ev (2002, 2007) are valid for arbitrary directed magnetic field.

Some words would be useful here about the simplifications in our method. First of all, we consider the optically thick plane plasma accretion disk neglecting the possible warps. Using the Milne problem we neglect the reflection of radiation from possibly existing central outflows (frequently this radiation lies far from observed optical wavelength bands). In all models of magnetized accretion disks, the solutions within the framework of the power-law dependence of magnetic field are sought inside the disk (see, for example, Pariev et al. 2003). Physically this assumption seems fairly natural if we remember that far from the sources the magnetic fields tend to dipole, quadrupole etc. forms, i.e. acquire the power-law dependence. We also assume that the radial dependence of the disk's magnetic field follows the same power-law in the range from the optical polarized emission down to the horizon. These simplifications now are commonly accepted, and can be considered as important assumptions of our theory.

The Milne problem in terms of vertical Thomson depth includes the possible vertical inhomogeneities of the atmosphere, so we do not include only possible horizontal inhomogeneities of the atmosphere. But if these inhomogeneities are smooth (with the characteristic length of many Thomson free lengths), the corrections should be neglected. In our paper we do not include the true absorption effects, considering the Milne problem in the limit of conservative atmosphere. Certainly, some our simplifications, such as the latter one, can easily be taken into account in the proposed method. We stress that this method can be generalized to more complex situations; in particular, it may be considered together with the other sources of polarized radiation (polar outflows, toroidal clumpy disks, etc.).

It should be mentioned that many AGNs models postulate the existence of a dusty geometrically thick obscuring region "the torus," which is placed far from the center of AGN (see Chang et al. 2007, and many references therein). This region give additional infrared radiation, as compared to the usual radiation of the interstellar medium. The spectrum of linear polarization from dusty media is characterized by Serkowski's formula (see Serkowski 1973; Martin 1989). If the spectrum of polarization of an AGN differs strongly (as in the source NGC 4258) from Serkowski's distribution, then the probability that the polarization comes from multiple scattering in plasma disk increases.

Our goal in this paper is to present the method of estimating of magnetic fields for fairly simple models. For this reason, in particular calculations we restrict ourselves to the most popular standard disk model of Shakura \& Sunyaev (1973).

\section{Basic equations}

We begin with the known expression for the Faraday rotation angle $\Psi$ at the Thomson optical path $\tau=\sigma_{\mathrm{T}} Z$ that is frequently used below:

$$
\begin{aligned}
\Psi & =\frac{\omega}{2 c}\left(n_{+}-n_{-}\right) Z=\frac{2 \pi N_{\mathrm{e}} e^{3} B_{z} Z}{m_{\mathrm{e}}^{2} c^{2} \omega^{2}} \equiv \frac{1}{2} \delta \tau \cos \theta \\
& \simeq 0.4\left(\frac{\lambda}{1 \mu \mathrm{m}}\right)^{2}\left(\frac{B}{1 G}\right) \tau \cos \theta,
\end{aligned}
$$

where $n_{ \pm}$are the refractive indices for right and left circular polarized electromagnetic waves, $\lambda=2 \pi c / \omega$ the wavelength of the radiation and $\theta$ the angle between the magnetic field $\boldsymbol{B}$ and line of sight $\boldsymbol{n}$ directions, $\sigma_{\mathrm{T}}$ Thomson cross-section, and $N_{\mathrm{e}}$ is the number density of electrons. The first expression in Eq. (1) is presented in many textbooks on optics. The second expression is derived in books on plasma physics (e.g. Kroll \& Trivelpiece 1973). This expression is usually used in radio astronomy (see Rohlfs \& Wilson 1996). In radiative transfer of optical radiation, the third expression in Eq. (1) is preferable, because it uses well known Thomson optical depth $\tau$ (see Dolginov et al. 1995; Gnedin \& Silant'ev 1997). According to Eq. (1) the Faraday dimensionless depolarization parameter $\delta$ takes a simple form:

$\delta=\frac{3}{4 \pi} \cdot \frac{\lambda}{r_{\mathrm{e}}} \cdot \frac{\omega_{B}}{\omega} \simeq 0.8 \lambda^{2}(\mu \mathrm{m}) B(G)$.

Here $\omega=2 \pi v=2 \pi c / \lambda$ is angular frequency, $\omega_{B}=e B / m_{\mathrm{e}} c$ the cyclotron frequency of an electron in a magnetic field, $r_{\mathrm{e}}=e^{2} / m_{\mathrm{e}} c^{2} \simeq 2.82 \times 10^{-13} \mathrm{~cm}$ is the classic electron radius. Parameter $\omega_{B} / \omega \simeq 0.93 \times 10^{-8} \lambda(\mu \mathrm{m}) B(G)$ is assumed to be small. This is the condition where the simple Eq. (1) is valid. In our paper we have $B \approx 10^{3}-10^{4} \mathrm{G}$ near the black hole's horizon. It this case the possible wavelength $\lambda$ can acquire values from X-rays up to infrared radiation with $\lambda \simeq 100 \mu \mathrm{m}$. Below we use the asymptotic formulae for the Stokes parameters, which are valid for $\Psi \geq 1$. Therefore, the wavelength range for which our model is valid extends from the X-ray band up to $\lambda \simeq 0.1 \mu \mathrm{m}$. Far from the horizon the magnetic field is less, and the depolarization parameter $\delta \ll 1$. In this case we have to use the usual Chandrasekhar formulae for polarization.

Silant'ev (2002) derived the asymptotical analytical formulae for the Stokes parameters of the radiation emitted from a magnetized, optically thick, plane-parallel atmosphere. For the Milne problem they are

$$
\begin{aligned}
I_{\lambda} & =\frac{F_{\lambda}}{2 \pi J_{1}} J(\mu), \\
Q_{\lambda} & =-\frac{F_{\lambda}}{2 \pi J_{1}} \frac{1-g}{1+g} \frac{\left(1-\mu^{2}\right)(1-k \mu)}{(1-k \mu)^{2}+(1-q)^{2} \delta^{2} \cos ^{2} \theta}, \\
U_{\lambda} & =-\frac{F_{\lambda}}{2 \pi J_{1}} \frac{1-g}{1+g} \frac{\left(1-\mu^{2}\right)(1-q) \delta \cos \theta}{(1-k \mu)^{2}+(1-q)^{2} \delta^{2} \cos ^{2} \theta},
\end{aligned}
$$

where $\mu=\cos i$ is the cosine of the angle between the normal to atmosphere $\boldsymbol{N}$ and the line of sight $\boldsymbol{n}, q$ the degree of true absorption $\left(q=\sigma_{\mathrm{a}} /\left(\sigma_{\mathrm{a}}+\sigma_{s}\right)\right)$, and $F_{\lambda}$ the total radiation flux. Function $J(\mu)$ describes the angular distribution of the radiation emerging from a disk. This function, as well as the numerical parameters $g, k$, and $J_{1}$ were tabulated by Silant'ev (2002). For electron conservative atmosphere $(q=0)$, the values of these parameters are $k=0, g=0.83255$, and $J_{1}=1.19402$. The Stokes parameters $Q$ and $U$ are given in the reference frame with the $\mathrm{X}$-axis lying on the plane $(\boldsymbol{n N})$ (see Fig. 1).

Formulae $(3,4)$ for polarization consider the last scattering of radiation before escaping from a semi-infinite magnetized atmosphere. For a high value of parameter $\delta$, the contribution of the secondary scattered photons is small because of the large Faraday depolarization. Even in the absence of a magnetic field, the main contribution to the polarization of emitted radiation comes from the last scattered photons. For this reason, Eqs. (3), (4) at the absence of magnetic field practically represent the classical Chandrasekhar-Sobolev polarization in the Milne problem (see, for example, Chandrasekhar 1950).

As far as the intensity of radiation $I(\mu)$ is concerned, one can remember (see Chandrasekhar 1950) that the polarization weakly influences the intensity. For Milne's problem without the 


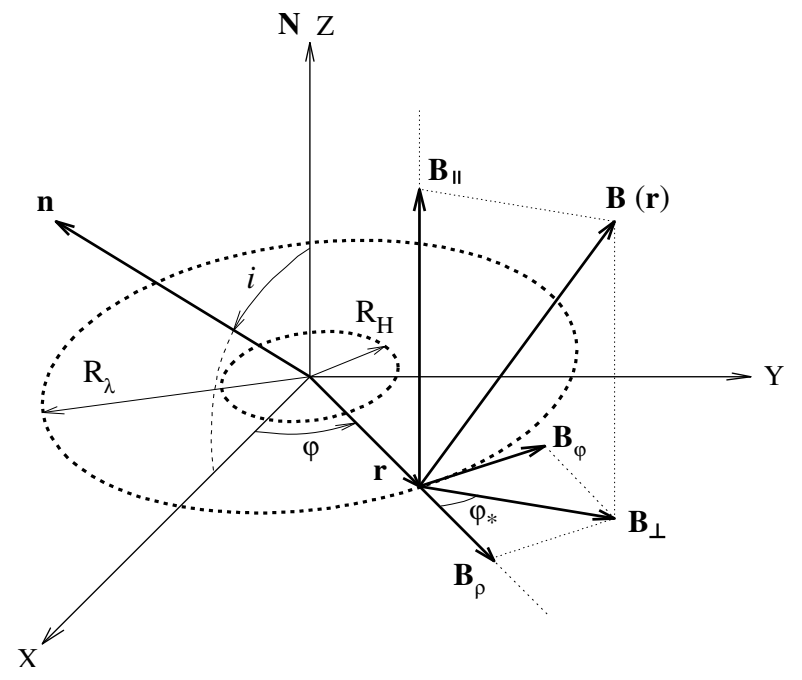

Fig. 1. Main notions of accretion disk surface, geometry of magnetic field $\boldsymbol{B}$, and line of sight $\boldsymbol{n}$ (see text).

true absorption $(q=0)$, we have $I(0) \sim 3.06$, whereas the separate transfer equation with the Rayleigh phase function gives $I(0) \sim 3.02$. For high values of $\delta$, the terms with Stokes parameters $Q$ and $U$ in the full system of transfer equations for parameters $I, Q$ and $U$ become very small $\sim 1 / \delta$, and they are negligible in the equation for intensity $I$. As a result, the radiation intensity obeys the separate transfer equation with the Rayleigh phase function (see, Silant'ev 1994 for more detail). Expression (3) presents the solution to this equation. For large $\delta$ the main contribution to polarization comes from to intensity term. Formulae (4) were obtained in this way.

Equations (2)-(4) allow us to derive the following approximate expressions for polarization degree $p(\boldsymbol{B}, \boldsymbol{n})$ and the position angle $\chi$ of radiation for an accreting magnetized disk:

$$
\begin{aligned}
p(\boldsymbol{B}, \boldsymbol{n}) & \simeq \frac{p(0, \mu)}{\sqrt{(1-k \mu)^{2}+(1-q)^{2} \delta^{2} \cos ^{2} \theta}}, \\
\tan 2 \chi & =\frac{U_{\lambda}}{Q_{\lambda}} \simeq \frac{(1-q) \delta}{1-k \mu} \cos \theta,
\end{aligned}
$$

where $B \cos \theta=\boldsymbol{B} \boldsymbol{n}$. Now we consider the case of dominant nonabsorbing electron scattering in accretion disks, i.e. $q=0$ and $k=0$. Photons escape the optically thick disk basically from the surface layer with $\tau \approx 1$. If the Faraday rotation angle $\Psi$ corresponding to this optical length becomes larger than unity, then the emerging radiation will be depolarized as a result of summarizing the radiation fluxes with the very different angles of Faraday rotation. Only for directions that are nearly perpendicular to the magnetic field are the Faraday rotation angles too small and depolarization does not occur. Certainly, the diffusion of radiation in the inner parts of a disk depolarizes the light, even in the absence of a magnetic field, because of multiple scattering of photons.

The existence of a magnetic field, hence Faraday rotation, only increases the depolarization process. It means that the polarization of outgoing radiation acquires a peak-like angular dependence with its maximum for perpendicular propagation. The sharpness of the peak increases with increasing magnetic field magnitude. The main region of allowed angles appears to be $\sim 1 / \delta$.

Another very important feature of the polarized radiation is the wavelength dependence of polarization degree $p$ and position angle $\chi$ which is very different from the case of classical electron scattering. This effect is briefly considered in Sect. 5. We consider the case where the Thomson cross-section does not depend on the radiation wavelength.

\subsection{Integral polarization from the accretion disk}

The axially symmetric accretion disks frequently are observed as a whole. The observed integral Stokes parameters $\langle Q\rangle$ and $\langle U\rangle$ are described by the azimuthally averaged formulae (3) and (4). To derive these expressions we introduce the following notions:

$\delta \cos \theta=\delta_{\|} \cos i+\delta_{\perp} \sin i \cos \left(\varphi+\varphi_{*}\right) \equiv a+b \cos \Phi$,

where $\varphi$ is the azimuthal angle of radius-vector $\boldsymbol{r}$ (the azimuthal angle of line of sight $\boldsymbol{n}$ is taken zero), $\varphi_{*}$ the angle between perpendicular magnetic field projection $\boldsymbol{B}_{\perp}=\left(B_{\rho}, B_{\varphi}\right)$, and the projection $\boldsymbol{B}_{\rho}\left(\cos \varphi_{*}=B_{\rho} / B_{\perp}\right)$ (see Fig. 1$)$. The value $B_{\|} \equiv B_{z}$ is the magnetic field projection along the normal $N$ to the accretion disk plane. According to Eqs. (1) and (6) we have

$a=\delta_{\|} \mu, \quad \delta_{\|}=0.8 \lambda^{2}(\mu \mathrm{m}) B_{\|}(G)$,
$b=\delta_{\perp} \sqrt{1-\mu^{2}}, \quad \delta_{\perp}=0.8 \lambda^{2}(\mu \mathrm{m}) B_{\perp}(G)$.

Using the axial symmetry of an accretion disk, we obtain (remember that $U(0, \mu) \equiv 0$ for Milne problem without magnetic field):

$$
\begin{aligned}
\langle Q\rangle= & Q(0, \mu) \\
& \times \frac{2}{\pi} \int_{0}^{\pi / 2} \mathrm{~d} \Phi \frac{1+a^{2}+b^{2} \cos ^{2} \Phi}{\left(1+a^{2}+b^{2} \cos ^{2} \Phi\right)^{2}-(2 a b \cos \Phi)^{2}}, \\
\langle U\rangle= & a Q(0, \mu) \\
& \times \frac{2}{\pi} \int_{0}^{\pi / 2} \mathrm{~d} \Phi \frac{1+a^{2}-b^{2} \cos ^{2} \Phi}{\left(1+a^{2}+b^{2} \cos ^{2} \Phi\right)^{2}-(2 a b \cos \Phi)^{2}} .
\end{aligned}
$$

The observed degree of the light polarization and the position angle are derived from parameters (8) in the usual way. For particular cases of pure normal $\left(\delta_{\perp}=0\right)$ and pure perpendicular $\left(\delta_{\|}=0\right)$ magnetic fields, expression (8) can be derived analytically. For the first case we have

$p(\boldsymbol{B}, \boldsymbol{n})=\frac{p(0, \mu)}{\sqrt{1+\delta_{\|}^{2} \mu^{2}}}, \quad \tan 2 \chi=\delta_{\|} \mu$.

For perpendicular magnetic field are there the formulae

$p(\boldsymbol{B}, \boldsymbol{n})=\frac{p(0, \mu)}{\sqrt{1+\delta_{\perp}^{2}\left(1-\mu^{2}\right)}}, \quad \chi \equiv 0$.

For a pure perpendicular magnetic field, the position angle $\chi=0$ comes from the axial symmetry of the problem, so the electric wave oscillations in this case occur parallel to the surface of an accretion disk. The case $\chi \neq 0$ can be realized if some $B_{z}$ component exists. It is interesting that the cases $\left(B_{\rho} \neq 0, B_{\phi}=0\right)$ and ( $B_{\rho}=0, B_{\phi} \neq 0$ ) give rise to the same formula (10). This is the consequence of the averaging procedure.

It is seen from Eqs. (8) that the relative degree of polarization $p(\boldsymbol{B}, \boldsymbol{n}) / p(0, \mu)$ and position angle $\chi$ only depend on dimensionless parameters $a$ and $b$, which are the functions of wavelength $\lambda$, magnetic fields $B_{\|}$or $B_{\perp}$, and inclination angle $i(\mu=\cos i)$. First we discuss the behavior of the relative degree of polarization and position angle on these two parameters. Remember that we consider conservative atmosphere with $q=0$, hence $k=0$. It is 
interesting to investigate how the polarization changes if we include the perpendicular magnetic field $B_{\perp}$ in the existing parallel magnetic field $B_{\|}$.

The average process takes the disk regions with the very different angles $\theta$ into account between the magnetic field $\boldsymbol{B}$ and the line of sight $\boldsymbol{n}$, and the integral polarization and position angle can acquire very different values. The numerical calculations show that position angle $\chi$ not only depends on parameter $a=\delta_{\|} \mu$ but also on the $b-$ parameter $\left(b=\delta_{\perp} \sqrt{1-\mu^{2}}\right)$. The existence of perpendicular magnetic field $\boldsymbol{B}_{\perp}$ diminishes the value of $\chi$ compared to the case of pure parallel magnetic field. This decrease is especially large if $b>a$ and the parameters $a$ and $b$ are close to unity. For $a \gg 1$ and $b<a$ the decrease of $\chi$ is small and practically $\chi \simeq 45^{\circ}$. But for $b>a \gg 1$, the position angle $\chi \rightarrow 0$. The special case is $a=b \gg 1$. In this case the position angle $\chi$ rapidly decreases from the limiting value $\sim 45^{\circ}$ for $a-b \simeq 1 \div 5$ to value $22.5^{\circ}$ at $b=a$, and then tends to zero for $b-a \simeq 1 \div 5$, so for large $a$ the intermediate values of $\chi$ can only occur in a rather narrow interval $b-a \simeq 1 \div 2$, i.e. at $a \simeq b$.

The degree of linear polarization $p$ depends on parameters $a$ and $b$ in a more complex form. For $a \leq 1$ the addition of the perpendicular magnetic field (parameter $b$ ) lowers the integral polarization. For $a>1$ there is the region of $b(b<a)$ where the polarization increases compared with the case of pure parallel magnetic field. The maximum polarization occurs at $b \simeq a$, and then the polarization decreases with the increase in parameter $b$. The increase in polarization at $b=a$ can be rather large. As a result, for value $a=b=5,10,20$, and 50 the relative polarization increase, as compared to purely parallel magnetic field, is equal to $160 \%, 224 \%, 317 \%$, and $504 \%$, respectively. It seems this effect stems some "resonant" regions in an accretion disk where the Faraday rotation from parallel magnetic field is balanced by opposite rotation from a perpendicular magnetic field. Of course, the magnitude of polarization decreases with the increase in $a$ and $b$. The numerical calculations demonstrate that the relative polarization degree $p(\boldsymbol{B}, \boldsymbol{n}) / p(0, \mu)$ is a symmetric function of parameters $a$ and $b$. The position angle $\chi$ does not possess this symmetry.

Now we shortly discuss the wavelength dependence of polarization degree $p(\lambda)$ and $\chi(\lambda)$, which follows from general formulae (8). More detailed discussion is presented in Sect. 5. For high values of parameters $a$ and (or) $b$ the spectra diminish $\sim 1 / \lambda^{2}$. But for the case $a=b$ mentioned above, the spectra diminish as $\sim 1 / \lambda$. Thus, the "resonant" effect also changes the asymptotic behavior of spectra. Note once more that this effect disappears beyond the interval $|a-b| \approx 1-5$. The spectra $\chi(\lambda)$ depend strongly on the relative value of the perpendicular magnetic field $B_{\perp}$ as compared to vertical component $B_{\|}$. If parameter $a \gg 1$ and $a \gg b$ the position angle $\chi(\lambda) \rightarrow 45^{\circ}$; i.e., it becomes independent of wavelength. For the "resonant" case $a=b$, this limiting value is equal to $22.5^{\circ}$.

The characteristic spectra of polarization and position angle as a function of inclination angle $i$ and magnetic fields $B_{\|}$and $B_{\perp}$ are presented in Fig. $2(\mu=\cos i)$. The mentioned case $a=b$ corresponds to $B_{\|} \mu=B_{\perp} \sqrt{1-\mu^{2}}$; i.e., at $B_{\|}=B_{\perp}$ it exists at $i=45^{\circ}$. For every $\lambda$ there exists its own value $a(\lambda)=b(\lambda)$, so, for $\lambda=1 \mu \mathrm{m}$ and $B_{\|}=B_{\perp}=5 \mathrm{G}$, this value is $a=b=2.828$. In this figure we present the relative polarization degree $p(\boldsymbol{B}, \boldsymbol{n}) / p(0, \mu)$ for the inclination angles $i=85^{\circ}, 60^{\circ}, 45^{\circ}$, and $30^{\circ}$. The values of the polarization degree $p(0, \mu)$ for these angles are equal to $7.80 \%, 2.25 \%, 1.08 \%$, and $0.43 \%$, respectively (see Chandrasekhar 1950). The presented spectra can help readers recognize general tendencies of polarization as a function of the basic system parameters.

We see that the higher magnetic field, the larger depolarization. For $i \approx 90^{\circ}$ and pure parallel magnetic field $\left(\boldsymbol{B}_{\perp}=0\right)$, the polarization degree tends to the Thomson value of polarization $p(0, \mu)$. This is quite natural because in these cases the magnetic field is practically perpendicular to the line of sight $\boldsymbol{n}$, and the Faraday rotation is low. The position angle $\chi$ is more sensitive to Faraday rotation, and tends to Thomson value $(\chi=0)$ slower than the polarization degree tends to the Thomson polarization. It is interesting that for this case the relative polarization degrees $p(\boldsymbol{B}, \boldsymbol{n}) / p(0, \mu)$ and position angles $\chi$ practically coincide for inclination angles $i=85^{\circ}$ and $i=30^{\circ}$, if the magnetic fields differ 10 times (for example, $B_{\|}=5$ and 50, or $B_{\|}=10$ and 100, etc.). This happens because the corresponding values of $\mu=\cos i$, 0.08715 and 0.86602 , differ approximately 10 times.

Comparing formulae (9) with (10), we find that the relative polarization degrees for perpendicular magnetic field $\left(B_{\|}=0\right)$ can be taken from the results drawn in Fig. $2 \mathrm{a}$, if one uses the substitution $i \rightarrow\left(90^{\circ}-i\right)$ there. The case $B_{\perp}=5 \mathrm{G}$ and $i=$ $60^{\circ}$ therefore coincides with the case $B_{\|}=5 \mathrm{G}$ and $i=30^{\circ}$. Of course, the position angle $\chi=0$ for perpendicular magnetic field.

For the case $B_{\|}=B_{\perp}$ (see Fig. 2b) the most depolarization occurs at $i \simeq 90^{\circ}$, in contrast to the pure parallel magnetic field. It is interesting that the relative degrees of polarization are the same for the inclinations $i$ and $90^{\circ}-i$. But the position angles are different in these cases. The relative polarization degree is higher for $i=45^{\circ}$ than for $i=30^{\circ}$, i.e. the change in this value is not monotonic. The case $i=45^{\circ}$ corresponds to equality $a=b \gg 1$. As mentioned above, in this case $\chi \rightarrow 22.5^{\circ}$. The righthand side of Fig. $2 b$ confirms this.

Figure $2 \mathrm{c}$ presents the spectra of the relative polarization degree and position angle for $B_{\|}=20 \mathrm{G}$ and $B_{\perp}=10 \mathrm{G}$ (the curves denoted by the usual numbers) and the opposite case $B_{\|}=10 \mathrm{G}$ and $B_{\perp}=20 \mathrm{G}$ (the curves denoted by numbers in brackets). The symmetry of relative polarization degree as a function of parameters $a$ and $b$ gives rise to the coincidence of spectra in the first case with those in the second case if the angle $i \rightarrow\left(90^{\circ}-i\right)$, so the relative polarization degree spectra at $30^{\circ}, 45^{\circ}, 60^{\circ}$ and $85^{\circ}$ coincide with the spectra denoted as $\left(60^{\circ}\right),\left(45^{\circ}\right),\left(30^{\circ}\right)$ and $\left(5^{\circ}\right)$, respectively. But the position-angle spectra are different for these two cases.

The spectra, presented in Fig. 2, demonstrate a large variability of values and forms for polarization degrees and position angles in the integral radiation escaping from the magnetized accretion disks.

\subsection{Some results from accretion disk models}

Models of a magnetic accretion disk with externally imposed, large- scale vertical magnetic field and anomalous magnetic field diffusion due to enhanced turbulent diffusion have been considered by Campbell (2000), Ogilvie \& Livio (2001), and Pariev et al. (2003).

We calculate the value of Faraday depolarization parameter $\delta$ for the model of an accreting disk suggesting the power-law radial dependence of the magnetic field:

$B(r)=B_{\mathrm{H}}\left(R_{\mathrm{H}} / r\right)^{n}$,

where $B(r)$ is the magnetic field inside an accretion disk, and $B_{\mathrm{H}}$ the magnetic field strength at the event horizon radius of 

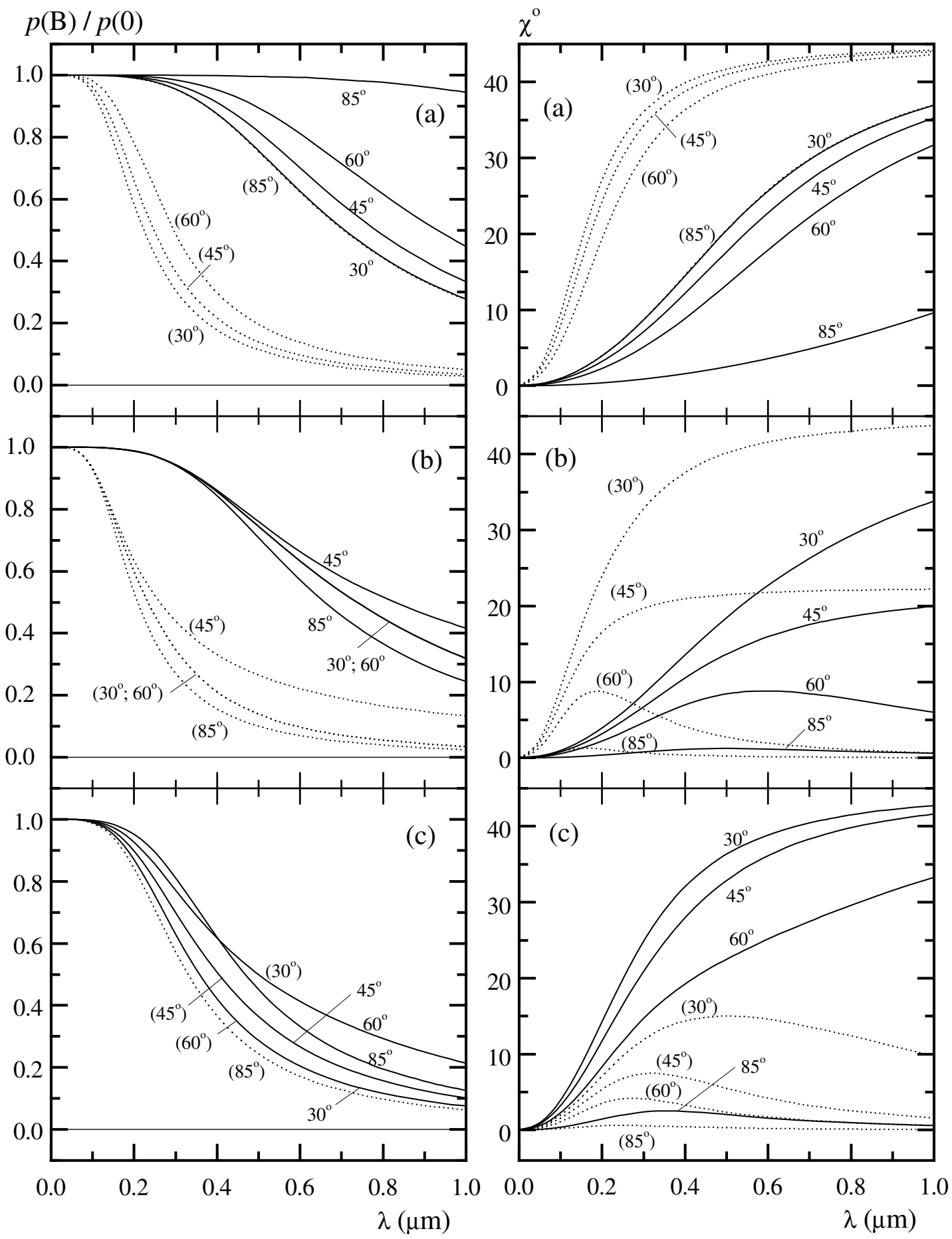

Fig. 2. Spectra of relative polarization degree $p(\boldsymbol{B}, \boldsymbol{n}) / p(0, \mu)$ and position angle $\chi$. The numbers denote the values of inclination angle $i$. The numbers in brackets refer to dotted curves. The a) figures demonstrate spectra for the pure parallel magnetic field with $B_{\|}=5 \mathrm{G}$ (bold curves) and $50 \mathrm{G}$ (dotted curves). The b) figures concern the case $B_{\|}=B_{\perp}=5 \mathrm{G}$ (bold curves) and $50 \mathrm{G}$ (dotted curves). The c) figures present spectra for $B_{\|}=20 \mathrm{G}$ and $B_{\perp}=10 \mathrm{G}$ (bold curves), and for $B_{\|}=10 \mathrm{G}$ and $B_{\perp}=20 \mathrm{G}$ (dotted curves).

a SMBH. The radius of the black hole horizon is (Novikov \& Thorne 1973)

$R_{\mathrm{H}}=\frac{G M_{\mathrm{BH}}}{c^{2}}\left(1+\sqrt{1-\left(a / M_{\mathrm{BH}}\right)^{2}}\right)$,

where $a / M_{\mathrm{BH}}$ is the spin of a Kerr black hole.

Pariev et al. (2003) have developed the detailed description of magnetized accretion disks with the different values for parameter $n$ (denoted as $\delta$ in their paper). The radial effective temperature dependence $T_{\mathrm{e}} \sim r^{-3 / 4}$ in their model is the same as in the Shakura-Sunyaev model. They find that, in the case of equipartition of magnetic pressure with radiation or thermal pressures, their results are close to Shakura-Sunyaev model with the viscosity parameter $\alpha=1$. As a most physically significant model, they investigate the case $n=5 / 4$ in more detail. Parameter $n$ must be greater than unity if we consider the disks with the diminishing gas density far from the black hole. It should also be noted that dependence (11) with $n=5 / 4$ takes 
place at the equipartition of magnetic energy with thermal and gravitational ones in the spherical accretion (see, for example, Melia 1992). Following to Pariev et al. (2003), we use mostly the case $n=5 / 4$.

The central problem is to derive the characteristic scale $R_{\lambda}$ that corresponds to the effective wavelength of polarimetric observations. At first glance, we can estimate the radius $R_{\lambda}$, suggesting that $\lambda=\lambda_{\mathrm{e}}$ corresponds to the value of rest-frame wavelength of the black body spectrum maximum,

$\lambda_{\mathrm{e}}=\frac{0.29}{T_{\mathrm{e}}(r)}, \quad \lambda_{0}=\lambda_{\mathrm{e}}(1+z)$,

where $\lambda_{0}$ is the wavelength in the observer system, and $z$ the cosmological redshift.

In a standard thin disk model (Shakura \& Sunyaev 1973), there are a black body radiation with an effective temperature profile of $T_{\mathrm{e}}=T_{\mathrm{H}}\left(r / R_{\mathrm{H}}\right)^{-3 / 4}$ and the scale length $R_{\lambda}$ defined by the point in the disk where the disk temperature matches the rest-frame wavelength of the monitoring band.

There is the series of papers where the semi-empirical method of determining of the accretion disk scale $R_{\lambda}$ has been developed (see Kochanek et al. 2006; Poindexter et al. 2008; Morgan et al. 2007, 2008). The authors used microlensing variability observed for gravitationally lensed quasars to find the accretion disk size and the observed (or rest-frame) wavelength relation. It is very important that the scaling appeared to be consistent with what is expected from the thin accretion disk theory of Shakura \& Sunyaev (1973).

This allows us to have the following size scaling (Poindexter et al. 2008):

$R_{\lambda}(\mathrm{cm})=0.97 \times 10^{10}\left(\frac{\lambda_{\mathrm{e}}}{\mu \mathrm{m}}\right)^{4 / 3}\left(\frac{M_{\mathrm{BH}}}{M_{\odot}}\right)^{2 / 3}\left(\frac{\eta}{\varepsilon}\right)^{1 / 3}$.

Here $R_{\lambda}$ is the distance in the accretion disk, which corresponds to $\lambda_{\mathrm{e}} ; \eta=L_{\mathrm{bol}} / L_{E \mathrm{dd}}, L_{\mathrm{Edd}}=1.3 \times 10^{38}\left(M_{\mathrm{BH}} / M_{\odot}\right) \mathrm{erg} \mathrm{s}^{-1}$ as the Eddington luminosity, and $\varepsilon$ as the rest-mass radiation conversion efficiency. Note that $R_{\lambda}$ does not depend on viscosity parameter $\alpha$. We use the commonly accepted relation between the bolometric luminosity $L_{\text {bol }}$ of the accretion disk and the accretion rate $\dot{M}$ :

$L_{\mathrm{bol}}=\varepsilon \dot{M} c^{2}$.

Theoretical calculations of parameter $\varepsilon$ depend on the details of the accepted model. Usually one presents the dependence of $\varepsilon$ on the spin parameter $a / M_{\mathrm{BH}}$ for every particular model. Naturally, this dependence is different for different models. Krolik (2007) present the comparison of $\varepsilon$-values from Novikov-Thorne model (Novikov \& Thorne 1973) and the model that take the jet luminosity into account. For spin parameter $a / M_{\mathrm{BH}}=0.5$, the first model gives $\varepsilon=0.081$, whereas the second model gives $\varepsilon=0.0063$. In some papers one considers $\varepsilon$ as an independent parameter in the model calculations. For example, Pariev $\&$ Colgate (2007) accept $\varepsilon=0.1, \eta=0.1$ and viscosity parameter $\alpha=0.01$.

The strong gravitational field near the black hole influences the Stokes parameters of outgoing radiation when they propagate to an observer. The detailed calculations of this effect have been done by Connors et al. (1980), Karas et al. (2004), and Dovciak et al. (2004). Usually these effects are important when describing X-ray emission from the vicinity of the black hole. The optical radiation arises far from this place, and we neglect these gravitational corrections.

\section{Magnetic field strength of NGC 4258}

NGC 4258 is a low-luminosity Seyfert II galaxy at the distance of about 7.2 Mpc, which harbors water masers (Modjaz et al. 2005). This object is usually considered as a very good laboratory for successfully measuring the magnetic field in accretion disk even very close to the central black hole. The spatial velocity distribution of water mega-maser sources in NGC 4258 on scales of $0.14-0.28 \mathrm{pc}$ indicates a thin Keplerian disk rotating around a black hole with a mass $M_{\mathrm{BH}}=3.9 \times 10^{7} M_{\odot}$ (Herrnstein et al. 1999). The accretion disk has an almost edge-on orientation with the radiation axis and its inclination angle is $i=83^{\circ} \pm 4^{\circ}$ (Pringle et al. 1999). A pc-scale jet closely aligns in projection on the sky with the rotation axis (Herrnstein et al. 1997).

Modjaz et al. (2005) present an analysis of polarimetric observations at $22 \mathrm{GHz}$ of the water vapor masers in NGC 4258 obtained with the VLA and the GBT. They do not detect any circular polarization in the spectrum indicative of Zeeman-induced splitting of the maser lines of water, and obtained only an upper limit on the magnetic field strengths. They obtained the 1- $\sigma$ upper limit value of the toroidal component of the magnetic field at a radius of $0.2 \mathrm{pc}$ the value of $90 \mathrm{mG}$ and determined a 1$\sigma$ upper limit of $30 \mathrm{mG}$ on the radial component at a radius of 0.14 pc. They also find from their observations of magnetic field limits that the geometrically thin disk model and the jetdisk model are better candidates for accounting for the extremely low-luminosity of NGC 4258.

More recently, Reynolds et al. (2008) have shown from analysis of SUZAKU and XMM-Newton observational data that the observed iron lines originate in the surface layers of an warped accretion disk at the distance $10^{3}-10^{4} R_{\mathrm{H}}$ from the black hole. In contrast to the majority of Seifert 2 galaxies, there was no indication of a Compton-thick obscuring torus. The weak iron line and the lack of a reflection point to circumnuclear environment that is remarkably clean of cold gas. They note that such a circumnuclear environment is only found in two AGNs - NGC 4258 and M81 that contrast to the majority of Seifert 2 galaxies.

This picture coincides with one by Herrnstein et al. (2005) pointed out earlier. According to them the observing intrinsic absorption in the X-ray spectrum can arise in the outer layers of the warped geometrically-thin accretion disk at the distance $\sim 29 \mathrm{pc}$ from the black hole, where the molecular-to-atomic transition occurs.

This picture allowed us to use our simple model of arising of optical polarized radiation without taking the warp contribution into account. Of course, the power-law dependence of the magnetic field is an important assumption in our derivation. This assumption is now commonly accepted (see Pariev et al. 2003).

The detected of polarized continuum and line emission from the nucleus of NGC 4258 was by Wilkes et al. (1995). After that, Barth et al. (1999) obtained spectropolarimetric observations of the NGC 4258 nucleus at the Keck II telescope. The observations were obtained on 1997 April 10 UT at the Keck II telescope with the LRIS spectropolarimeter. The results of these observations are presented in the Table 1 of the paper by Barth et al. (1999). For the continuum polarization they obtained the following results:

$$
\begin{aligned}
& p(\lambda \lambda 4000 \div 4800 \dot{A})=0.38 \pm 0.03 \%, \chi=12^{\circ} \pm 2^{\circ} \\
& p(\lambda \lambda 5100 \div 6100 \dot{A})=0.35 \pm 0.01 \%, \chi=7^{\circ} \pm 1^{\circ} \\
& p(\lambda \lambda 7500 \div 8500 \dot{A})=0.29 \pm 0.02 \%, \chi=8^{\circ} \pm 2^{\circ}
\end{aligned}
$$

We see that the polarization is weakly increasing to the short wavelength range, but the value of a position angle is practically 
constant. The position angles $\chi$ are the angles between wave electric field oscillations and the surface of the accretion disk.

\subsection{Estimates of magnetic field in the model of nonturbulent accretion disk}

For the inclination angle $i=83^{\circ}$, the expected polarization should have the value $p(0, \mu)=6.9 \%(\mu=\cos i=0.122)$. From Eqs. (9) and (10) for degree of polarization we find that possible parameters $a=\delta_{\|} \mu$ and $b=\delta_{\perp} \sqrt{1-\mu^{2}}$ are near the value 20 . The position angle $\chi$ for this value of $a$ is equal to $43.6^{\circ}$, which is far from observing values (see Eq. (21)). From the discussion in Sect. 2.1 we know that for high values of parameters $a$ and $b$, the possibility of small position angles exists if $a \simeq b$.

The exact formulae (8) give us the values $a=122$ and $b=122.9$, which correspond to observed values of polarization degree $0.38 \%$ and $\chi=12^{\circ}$ at $\lambda=0.44 \mu \mathrm{m}$. These values correspond to $\delta_{\|}=1000$ (or $B_{\|}=6400 \mathrm{G}$ ) and $\delta_{\perp}=124$ (or $B_{\perp}=800 \mathrm{G}$ ). The analogous values of parameters for the polarization degree $0.35 \%$ and $\chi=7^{\circ}$ at $\lambda=0.56 \mu \mathrm{m}$ are $\delta_{\|}=762.3$ (or $B_{\|}=3037 \mathrm{G}$ ) and $\delta_{\perp}=95.5$ (or $B_{\perp}=380.5 \mathrm{G}$ ). For the case $\lambda=0.8 \mu \mathrm{m}\left(p=0.29 \%, \chi=8^{\circ}\right)$, the formulae (8) give $B_{\|}=2401.4 \mathrm{G}$ and $B_{\perp}=298.3 \mathrm{G}$. We see that in all cases the normal magnetic field $B_{\|}$is greater than $B_{\perp}$. Because the effective temperature $T_{\mathrm{e}}$ decreases with the increase in the distance from the inner radius of an accretion disk, we conclude that magnetic field also decreases with the growing distance.

What seems unsatisfactory in these results is their sensitivity to small variations in parameters $a$ and $b$. If these parameters change their values $(\Delta(b-a) \simeq 1)$ slightly the solution is impossible. For magnetic fields it means that the values have not to change its values greater than $10 \mathrm{G}$. This is very improbable for real situations in accretion disks. For this reason we have to seek a more satisfactory model where this sensitivity does not occur. Such a model really exists. It takes into account that the magnetic field can be turbulent.

\subsection{Estimates of magnetic field in the model of turbulent accretion disk}

According to Silant'ev $(2005,2007)$ the chaotic component $\boldsymbol{B}^{\prime}$ of the magnetic field $\left(\boldsymbol{B}=\boldsymbol{B}_{0}+\boldsymbol{B}^{\prime}\right)$, where $\boldsymbol{B}_{0}$ is a regular part of the magnetic field, gives rise to additional extinction of the intensity of linearly polarized waves (parameters $Q$ and $U$ ) due to small scale chaotic Faraday rotations. The Gaussian distribution of turbulent velocities was assumed. Mathematically, this effect is analogous to the known problem of diffusion of scalar impurity in a stochastic velocity field (see, for example, van Kampen 1981). In our case, the Faraday rotation angle replaces the role of impurity. The main part of the effective cross-section $\sigma_{*} \equiv \sigma_{\mathrm{T}} C$ corresponding to this additional extinction, takes a very simple form:

$C=f_{B} \tau_{1}\left\langle\delta^{\prime 2}\right\rangle \simeq 0.64 f_{B} \tau_{1} \lambda^{4}(\mu \mathrm{m})\left\langle B^{\prime 2}(G)\right\rangle / 3$

Here, $\tau_{1}$ is mean Thomson optical radius of turbulent eddies, $f_{B} \simeq 1$ is a constant characterizing a particular form of two-point turbulent velocity correlations (for estimations we take $f_{B}=1$ ), and $\delta^{\prime}$ and $\boldsymbol{B}^{\prime}$ are fluctuating parts of Faraday depolarization parameter $\delta$ and magnetic field $\boldsymbol{B}$, respectively. Clearly, the additional extinction should be proportional to the mean square of fluctuations of physical parameter $\delta$. Because $\delta \sim \lambda^{2}$, the dimensionless parameter $C \sim \lambda^{4}$.
The asymptotic formulae taking this effect into account have the same form as formulae (3-5) with the substitution $(1-k \mu) \rightarrow$ $(1+C)$. (Remember that we consider the conservative atmosphere with $q=k=0$.) In particular, the formulae (8) acquire the form

$$
\begin{aligned}
&\langle Q\rangle=(1+C) Q(0, \mu) \\
& \times \frac{2}{\pi} \int_{0}^{\pi / 2} \mathrm{~d} \Phi \frac{(1+C)^{2}+a^{2}+b^{2} \cos ^{2} \Phi}{\left[(1+C)^{2}+a^{2}+b^{2} \cos ^{2} \Phi\right]^{2}-(2 a b \cos \Phi)^{2}} \\
&\langle U\rangle=a Q(0, \mu) \\
& \quad \times \frac{2}{\pi} \int_{0}^{\pi / 2} \mathrm{~d} \Phi \frac{(1+C)^{2}+a^{2}-b^{2} \cos ^{2} \Phi}{\left[(1+C)^{2}+a^{2}+b^{2} \cos ^{2} \Phi\right]^{2}-(2 a b \cos \Phi)^{2}} \cdot
\end{aligned}
$$

Here parameters $a$ and $b$ are presented by formulae (7), where $B_{\|}$ and $B_{\perp}$ denote the regular (mean) values of corresponding magnetic fields, i. e. $B_{0 \|}$ and $B_{0 \perp}$. The numerical calculations show that the relative polarization degree $p(\boldsymbol{B}, \boldsymbol{n}) / p(0, \mu)$ is also the symmetric function of parameters $a$ and $b$, as for the nonturbulent atmosphere.

The parameter $C$ is fairly large for our case, $C \approx 10 \div 20$. In this case the term $(2 a b \cos \Phi)^{2}$ in denominators of formulae (18) can be neglected and we obtain the following analytical expressions:

$$
\begin{aligned}
\tan 2 \chi & \cong \frac{a}{(1+C)(1+d)}, \quad d=\frac{b^{2}}{(1+C)^{2}+a^{2}}, \\
p & \cong \frac{p(0, \mu)}{\left[(1+C)^{2}+a^{2}\right] \sqrt{(1+d)}} \sqrt{(1+C)^{2}+\frac{a^{2}}{(1+d)^{2}}}
\end{aligned}
$$

These expressions are fairly exact up to $a, b<C$. If parameters $a$ or $b$ are equal to zero, formulae (19) are exact. In particular, for the cases of pure normal regular magnetic field $\boldsymbol{B}_{0 \perp}=0$ $(b=0)$, and pure perpendicular regular field $\boldsymbol{B}_{0 \|}=0(a=0)$, they transform to the following exact formulae:

$$
\begin{aligned}
& p(\boldsymbol{B}, \boldsymbol{n})=\frac{p(0, \mu)}{\sqrt{(1+C)^{2}+\delta_{\|}^{2} \mu^{2}}}, \quad \tan 2 \chi=\frac{\delta_{\|} \mu}{1+C}, \\
& p(\boldsymbol{B}, \boldsymbol{n})=\frac{p(0, \mu)}{\sqrt{(1+C)^{2}+\delta_{\perp}^{2}\left(1-\mu^{2}\right)}}, \quad \chi \equiv 0 .
\end{aligned}
$$

In the limit case of nonturbulent magnetic field $(C=0)$, they coincide with the formulae (9) and (10). If the chaotic magnetic field $B^{\prime}$ is fairly high $\left(\tau_{1} \lambda^{2}(\mu \mathrm{m})\left\langle B^{\prime 2}\right\rangle \geq 4 B_{0 \|, 0 \perp}\right.$, see Silant'ev $2007)$, then the depolarization of radiation is due to additional extinction (17): $p\left(B^{\prime}, \boldsymbol{n}\right)=p(0, \mu) / C \sim 1 / \lambda^{4}\left\langle B^{\prime 2}\right\rangle$. If we suppose that $\left\langle B^{\prime 2}\right\rangle=$ const., then $p(\lambda)_{\text {turb }} \sim \lambda^{-4}$.

The existence of new parameter $C$ describing the level of magnetic field fluctuations makes the estimation of mean values of $B_{\|}$and $B_{\perp}$ difficult. In our case this problem is simpler because the level of magnetic fluctuations (parameter $C$ ) changes slowly with the variations in parameters $a$ and $b$. Indeed, for the pure normal magnetic field $(b=0)$ we found $a=7.37, C=15.5$ for $\lambda=0.44 \mu \mathrm{m} ; a=4.77, C=18.13$ for $\lambda=0.56 \mu \mathrm{m}$, and $a=6.56, C=21.88$ at $\lambda=0.8 \mu \mathrm{m}$. In the limiting case of high values of polarization degree $(a=b)$, these values are: $a=b=$ 8, $C=14 ; a=b=5, C=17.5$, and $a=b=7, C=21$, respectively; i.e., parameter $C$ is practically the same for these two cases.

For this reason we calculate the $a$ and $b$ parameters using the mean values for parameter $C$; i.e., $C=14.75,17.81$ 
and 21.44, respectively. This gives the values $a=8, b=6$ $\left(B_{\|}=423 G, B_{\perp}=39 \mathrm{G}\right)$ for $\lambda=0.44 \mu \mathrm{m}$; for $\lambda=0.56 \mu \mathrm{m}-$ $a=5, b=3.5\left(B_{\|}=163 \mathrm{G}, B_{\perp}=14 \mathrm{G}\right)$; and for $\lambda=0.8 \mu \mathrm{m}-$ $a=7, b=5.5\left(B_{\|}=112 \mathrm{G}, B_{\perp}=10.8 \mathrm{G}\right)$. These values of magnetic fields are lowere than those for the case of nonturbulent accretion disk. But important is that they were derived without restriction $a \simeq b$.

Let us estimate the level of magnetic fluctuations taking the mean values for parameter $C$. We also assume that $f_{B}=1$ and $\tau_{1}=0.1$. We do not know the real distribution of turbulent eddies in a turbulent accretion disk. The general picture of turbulence consists of cascade of eddies with different dimensions. The small eddies with $\tau_{1} \ll 1$ includes a value propto a parameter $C$. The large eddies do not allow us to describe the considered effect in the range of radiative transfer equations. It seems that our value $\tau_{1} \simeq 0.1$ is fairly natural for describing the turbulent effects in magnetized plasma. If we increase $\tau_{1}$ to $k$-times, then the level of magnetic fluctuations $\left\langle B^{\prime 2}\right\rangle$ decreases to k-times. The real value of parameter $\tau_{1}$ can therefore be estimated by independent estimation of magnetic fluctuations. After that we obtain the values $B^{\prime} \approx 136 G, 92 \mathrm{G}$, and $49 \mathrm{G}$ for the mean square root values of magnetic fluctuations at places where the thermal radiation has a maximum for $\lambda=0.44 \mu \mathrm{m}, 0.56 \mu \mathrm{m}$, and $0.8 \mu \mathrm{m}$, respectively. These values of fluctuations are equal to $31 \%, 56 \%$, and $44 \%$ of the mean magnetic fields for mentioned wavelengths.

\subsection{Estimates of the magnetic field at the horizon of the black hole in NGC 4258}

We now proceed to the estimation of the magnetic field strength in NGC 4258 using the maser polarimetric data. The magnetic field structures in accretion disks are difficult to observe and remain poorly known. If the disk is penetrated by a dipole field of the central object or by a global field of the surrounding interstellar medium, there may be a net vertical flux. Sano et al. (2004) consider the models of an accretion disk with a uniform magnetic field. The stress forces in accretion disks may be proportional to $B_{z}$ (Hawley et al. 1995) or to $B_{z}^{2}$ (Turner et al. 2003).

Zhang \& Dai (2008) have studied the effect of a global magnetic field on viscously rotating and vertically integrated accretion disks around compact objects using a self-similar treatment. They show that the strong magnetic field in the vertical direction prevents the disk from being accreted and decreases the effect of the gas pressure.

On the other hand, Königl (1989) and Cao (1997) underline that the inclination of the field lines at the surface of the disk plays a crucial role in the magnetically driven outflow. They show that, for the nonrelativistic case, a centrifugally driven outflow of matter from the disk is only possible if the poloidal component of the magnetic field makes an angle less than a critical $60^{\circ}$ with the disk surface.

Now let us estimate the value of $R_{\lambda}$ for the model of a standard accretion disk using the observational data of NGC 4258. The spatial structure of standard accretion disk have been calculated by Poindexter et al. (2008). The size scaling is determined by Eq. (14). The basic physical parameters of the central nucleus of NGC 4258 are $M_{\mathrm{BH}}=3.9 \times 10^{7} M_{\odot}$ and $\eta=L_{\text {bol }} / L_{\mathrm{Edd}}=$ $10^{-2.27}=0.0054$ (Satyapal et al. 2005). We use these estimations below in numerical calculations.
The estimates of the scaleradius $R_{\lambda}$ from Eq. (14) give the following results:

$R_{\lambda}=1.38 \times 10^{14}\left(\frac{0.1}{\varepsilon}\right)^{1 / 3} \mathrm{~cm}, \lambda_{\text {rest }}=0.44 \mu \mathrm{m}$,

$R_{\lambda}=1.9 \times 10^{14}\left(\frac{0.1}{\varepsilon}\right)^{1 / 3} \mathrm{~cm}, \lambda_{\text {rest }}=0.56 \mu \mathrm{m}$,

$R_{\lambda}=3.06 \times 10^{14}\left(\frac{0.1}{\varepsilon}\right)^{1 / 3} \mathrm{~cm}, \lambda_{\text {rest }}=0.8 \mu \mathrm{m}$.

For $\varepsilon=0.1$, which corresponds to $a / M_{\mathrm{BH}} \simeq 0.6$ in the model of Novikov \& Thorne (1973), the ratios of $R_{\lambda} / R_{\mathrm{H}}$ are equal to

$R_{\lambda}(0.44) / R_{\mathrm{H}}=13 ; R_{\lambda}(0.56) / R_{\mathrm{H}}=18 ;$

$R_{\lambda}(0.80) / R_{\mathrm{H}}=29$.

The formulae (21) for arbitrary wavelength $\lambda$ acquire the form:

$R_{\lambda}=1.94 \times 10^{14} \frac{\lambda^{4 / 3}}{\varepsilon^{1 / 3}}$.

These estimates show that $R_{\lambda}<R_{a b}$, if the viscosity parameter $\alpha \geq 0.1$ (see Eq. (23)). Here $R_{a b}$ is the boundary radius between two zones of the standard accretion disk (Shakura \& Sunyaev 1973): (a) a radiation pressure-dominated zone and (b) a gas pressure-dominated zone. In both zones the opacity comes from Thomson scattering. The boundary between these zones is given by Shakura \& Sunyaev (1973) and Pariev \& Colgate (2007):

$R_{a b}=236 R_{\mathrm{H}}\left(\frac{\alpha}{0.01}\right)^{2 / 21}\left(\frac{M_{\mathrm{BH}}}{10^{8} M_{\odot}}\right)^{2 / 21}\left(\frac{\eta}{\varepsilon}\right)^{16 / 21}$.

This means that the characteristic spatial radius of an accretion disk $R_{\lambda}$, corresponding to observed wavelength $\lambda$, lies for NGC 4258 in the zone (a). For the frequently used value $\alpha=$ 0.01 (see Pariev \& Colgate 2007), we have $R_{a b}=23.2 R_{\mathrm{H}}$ at $\epsilon=0.1$; i.e., the inequality $R_{\lambda}<R_{a b}$ takes place only for $\lambda=0.44 \mu \mathrm{m}$ and $0.56 \mu \mathrm{m}$.

We use the polarimetric data by Modjaz et al. (2005). These data allow a $1-\sigma$ upper limit of $B_{\text {mas }} \sim 30 \mathrm{mG}$ on the radial component of the disk magnetic field at the radius of $0.14 \mathrm{pc}$. Using the power-law radial dependence of magnetic field (11), we obtain the following expression:

$B_{\text {mas }}=B\left(R_{\lambda}\right)\left(\frac{R_{\lambda}}{R_{\text {mas }}}\right)^{5 / 4}=0.018\left(\frac{0.1}{\varepsilon}\right)^{5 / 12}$

where $R_{\text {mas }}=0.14 \mathrm{pc}, B\left(R_{\lambda}\right)=425 \mathrm{G}$ for $\lambda=0.44 \mu \mathrm{m}$. This expression can be used for a crude estimate of parameter $\varepsilon$. In our case we obtain $\varepsilon \approx 0.03$. In this case, $R_{a b}=58.2 R_{\mathrm{H}}(\alpha / 0.01)^{2 / 21}$. This means that inequality $R_{\lambda}<R_{a b}$ occurs even for value $\alpha=0.01$, mentioned above. The values $R_{\lambda}$ for $\epsilon=0.03$ are higher than values (22) to 1.5 times, i. e. they also lesser than $R_{a b}$, if we take $\alpha=0.01$.

Finally, we can estimate the magnetic field strength $B_{\mathrm{H}}$ at the horizon radius of the black hole in NGC 4258 using the data for $R_{\lambda} / R_{\mathrm{H}}$ presented in expressions (22), and for our values $B\left(R_{\lambda}\right)$ for the turbulent accretion disk model (see Sect. 3.2). Taking in Eq. (11) $r=R_{\lambda}$, we obtain the expression:

$B_{\mathrm{H}}=B\left(R_{\lambda}\right)\left(\frac{R_{\lambda}}{R_{\mathrm{H}}}\right)^{5 / 4}(\mathrm{G})$.

As a result, we obtain for $\lambda=0.44 \mu \mathrm{m}, 0.56 \mu \mathrm{m}$, and $\lambda=$ $0.8 \mu \mathrm{m}$ the following values $B_{\mathrm{H}}=1.05 \times 10^{4} \mathrm{G}, 6.06 \times 10^{3} \mathrm{G}$ 
Table 1. The value of $B_{\mathrm{H}}[\mathrm{G}]$ for various data of Kerr parameter and radiative efficiency.

\begin{tabular}{lcrrr}
\hline \hline Source & $\delta_{\|} ; \delta_{\perp}$ & $\frac{a}{M_{\mathrm{BH}}}=0.5$ & $\frac{a}{M_{\mathrm{BH}}}=0.998$ & $\frac{a}{M_{\mathrm{BH}}}=-0.9$ \\
& & $\varepsilon=0.081$ & $\varepsilon=0.32$ & $\varepsilon=0.039$ \\
\hline NGC 4258 & $41 ; 3.5$ & $6.6 \times 10^{3}$ & $4 \times 10^{3}$ & $9.6 \times 10^{3}$ \\
\hline
\end{tabular}

and $7.57 \times 10^{3} \mathrm{G}$, respectively (at $\varepsilon=0.1$, which corresponds to spin (Kerr) parameter $a / M_{\mathrm{BH}} \simeq 0.6$ in the model of Novikov $\&$ Thorne 1973). At $\epsilon=0.03$, these values are to 1.66 times higher. This case approximately corresponds to $a / M_{\mathrm{BH}} \approx-0.95$ (see Krolik 2007).

Our estimates are slightly different as a result of different error intervals of polarimetric data (see Eq. (16)). Besides, some uncertainty exists in the choice of the level of fluctuations (parameter $\mathrm{C}$ ). It seems for $\lambda=0.56 \mu \mathrm{m}$ that this uncertainty is less than for other wavelengths. For this reason the estimate $B_{\mathrm{H}}=6.06 \times 10^{3} \mathrm{G}$ seems to be preferable. As an example, we present the estimates for this effective wavelength and other various parameters $\varepsilon$ and $a / M_{\mathrm{BH}}$ in Table 1 .

In the estimations, presented above we take $n=5 / 4$ in the basic Eq. (11). How do we change the estimations for other values of $n$ ? The calculations give the following values of $B_{\mathrm{H}}$ at the event horizon of the supermassive black hole in NGC 4258: $B_{\mathrm{H}}=3 \times 10^{3} \mathrm{G}$ at $n=1$, and $B_{\mathrm{H}}=5.3 \times 10^{4} \mathrm{G}$ at $n=2$. These results indicate that the magnetic field strength of SMBH in NGC 4258 at the event horizon should be at the level $\approx 10^{3}-10^{4} \mathrm{G}$.

It should be noted that our estimations do not use the values of viscosity parameter $\alpha$. It was only shown that, for $\alpha>0.1$, the radius $R_{\lambda}$ lies inside zone (a). Of course, our estimations depend on parameters $\varepsilon$ and the power-law index $n$, which, in principle, are to be found in a detailed model of an accretion disk.

The data in Pariev et al. (2003) are given for $r>2.5 r_{\mathrm{g}}\left(r_{\mathrm{g}}=\right.$ $2 G M_{\mathrm{BH}} / c^{2}$ is the gravitational radius of black hole), i. e. slightly beyond the horizon radius $R_{\mathrm{H}}$ (beyond $2.2 R_{\mathrm{H}}$ for $\varepsilon=0.1$, and beyond $5 R_{\mathrm{H}}$ for $\varepsilon=0.03$ ). It seems that they used the simplified theory, which do not "work" near the horizon. The calculations in Pariev et al. (2003) correspond to magnetic dominated regime; i.e., magnetic energy is greater than radiative thermal energy. Because $R_{\lambda}$ lies inside the zone (a) (radiation dominated zone), we derive that plasma parameter $\beta=P_{\text {gas }} / P_{\text {magn }}<1$ in model of Pariev et al. (2003). Here $P_{\text {gas }}$ and $P_{\text {magn }}$ are gas and magnetic pressures, respectively.

\section{Magnetic coupling process in AGN and QSO: testing by continuum polarization}

Li (2002), Wang et al. (2002, 2003), Zhang et al. (2005) studied the magnetic coupling process (MC) as an effective mechanism for transferring energy and angular momentum from a rotating black hole to its surrounding accretion disk. This process can be considered as one of the variants of the Blandford-Znajek (BZ) process (Blandford \& Znajek 1977; Blandford \& Payne 1982). It is assumed that the disk is stable, perfectly conducting, thin, and Keplerian. The magnetic field is assumed to be constant on the black hole horizon and to vary as a power law with the radius of the accretion disk.

Since the magnetic field on the horizon $B_{\mathrm{H}}$ is brought and held by its surrounding magnetized matter of a disk, the some relation must exist between the magnetic field strength and accretion disk and, finally, the bolometric luminosity of AGN (see Ma et al. 2007). This relation takes a form

$B_{\mathrm{H}}(G)=\frac{k^{1 / 2}\left(2 L_{\mathrm{bol}} / \varepsilon c\right)^{1 / 2}}{R_{\mathrm{H}}}$.

Here $L_{\mathrm{bol}}=\varepsilon \dot{M} c^{2}, R_{\mathrm{H}}$ is the horizon radius (see Eq. (12)), $\dot{M}$ is the accretion rate, $\varepsilon$ the radiative efficiency (calculated, for example, by Novikov \& Thorne 1973; Krolik 2007; Shapiro 2007, see also Sect. 2.2). Coefficient $k$ is the inverse plasma parameter $\beta=P_{\text {gas }} / P_{\text {magn }}$, where $P_{\text {gas }}$ and $P_{\text {magn }}$ are gas and magnetic pressures, respectively. We shall consider the case $\beta=1(k=1)$ in future, i.e. the equipartition case.

Then Eq. (27) is transformed into

$B_{\mathrm{H}}(G)=\left(\frac{M_{\odot}}{M_{\mathrm{BH}}}\right)^{1 / 2}\left(\frac{\eta}{\varepsilon}\right)^{1 / 2} \frac{6.2 \times 10^{8}}{1+\sqrt{1-\left(a / M_{\mathrm{BH}}\right)^{2}}}$,

where $a / M_{\mathrm{BH}}$ is the Kerr parameter, $\eta=L_{\mathrm{bol}} / L_{\mathrm{Edd}}$ and $L_{\mathrm{Edd}}=$ $1.3 \times 10^{38}\left(M_{\mathrm{BH}} / M_{\odot}\right)\left(\mathrm{erg} \mathrm{s}^{-1}\right)$ is the Eddington luminosity.

We next calculate the expected polarization value of radiation in a number of specific AGNs, taking the effect of Faraday depolarization into account, as considered above. In mechanisms of magnetic coupling, we mainly consider the case where magnetic field is supposed directed along the normal to the accretion disk (see, for example, Wang et al. 2002; Ma et al. 2007). Our estimates of the magnetic field in NGC 4258 (see Sect. 3.2) show that the vertical magnetic field is much larger than the perpendicular one. For these reasons, we also consider only $\boldsymbol{B}_{\|}$fields as first approximation, so we have to determine $B_{\|}=B\left(R_{\lambda}\right)$ from Eq. (25). The values $R_{\mathrm{H}}$ and $R_{\lambda}$ are presented in Eqs. (12) and (14), and $B_{\mathrm{H}}-$ in Eq. (28). As a result, we obtain the following formula for dimensionless parameter $\delta_{\|}$:

$$
\begin{aligned}
\delta_{\|}= & 0.8 \lambda^{2}(\mu \mathrm{m}) B_{\|}(G) \simeq 474 \lambda^{1 / 3}(\mu \mathrm{m})\left(\frac{\eta}{\varepsilon}\right)^{1 / 12} \\
& \times\left(\frac{M_{\mathrm{BH}}}{M_{\odot}}\right)^{-1 / 12}\left(1+\sqrt{1-\left(\frac{a}{M_{\mathrm{BH}}}\right)^{2}}\right)^{1 / 4}
\end{aligned}
$$

The estimations of polarization degree $p(\lambda)$ can be obtained from Eq. (9).

A systematic analysis of a large sample of AGN available in the BeppoSAX public archive was performed by Grandi et al. (2006). Their sample includes AGN of various types. Narrow line radio galaxies (NLRG), broad line radio galaxies (BLRG), steep spectrum radio quasars (SSRQ) and flat spectrum radio quasars (FSRQ) (see Table 6 from their paper, where the values $M_{\mathrm{BH}} / M_{\odot}$ and $\eta=L_{\mathrm{bol}} / L_{\text {edd }}$ are presented.

The results of our calculations of the dimensionless depolarization parameter $\delta_{\|}$are

BLRG : $\delta_{\|} \simeq 79 ; 60 ; \quad \mathrm{NGRG}: \delta_{\|} \simeq 67 ; 57$,

SSRQ : $\delta_{\|} \simeq 79 ; 60 ;$ FSRQ $: \delta_{\|} \simeq 108 ; 82$,

Seyfert $1: \delta_{\|} \simeq 125 ; 95$,

where the first numbers correspond to $\varepsilon=0.081$ and $a / M_{\mathrm{BH}}=$ 0.5 , and the second ones correspond to $\varepsilon=0.32, a / M_{\mathrm{BH}}=$ 0.998 .

The estimations of possible $p(\mu)$ for the inclination angle of the accretion disk $i=60^{\circ}(\mu=\cos i, p(0, \mu)=2.25 \%)$ are presented in Table 2. Because all values of $\delta_{\|} \gg 1$, we can use the approximate formula $p(\mu) \simeq p(0, \mu) / \delta_{\|} \mu$. This formula is valid up to $i=87^{\circ}$, so for $i=85^{\circ}(p(0, \mu) \simeq 7.8 \%, \mu=0.087)$ the 
Table 2. The value of linear polarization from accretion disks.

\begin{tabular}{|c|c|c|c|c|}
\hline Source & $\log \frac{M_{\mathrm{BH}}}{M_{\odot}}$ & $\log \frac{L_{\mathrm{bol}}}{L_{\mathrm{Edd}}}$ & $\begin{array}{c}p[\%] \\
\frac{a}{M_{\mathrm{BH}}}=0.5 \\
\varepsilon=0.081\end{array}$ & $\begin{array}{c}p[\%] \\
\frac{a}{M_{\mathrm{BH}}}=0.998 \\
\varepsilon=0.32\end{array}$ \\
\hline$\overline{B L R G}$ & 8.64 & -1.5 & 0.06 & 0.057 \\
\hline NLRG & 8.14 & -2.92 & 0.09 & 0.067 \\
\hline SSRQ & 9.29 & -0.90 & 0.05 & 0.057 \\
\hline FSRQ & 9.01 & 0.44 & 0.94 & 0.042 \\
\hline Seyfert 1 & 7.23 & -0.59 & 1.40 & 0.036 \\
\hline
\end{tabular}

polarization degree is to 20 times higher than values presented in Table $2(p(\mu=0.087)=(p(0,0.087) / p(0,0.5))(0.5 / 0.087) \simeq$ $20)$. For other inclination angles $i$, the calculations are analogous. We stress that polarization observations of sources, presented in Table 2, do not exist up to now, so we present only possible values of polarization degrees. This procedure, presented below, can be used to estimate the source parameters if the polarization data are available.

The limiting case $a / M_{\mathrm{BH}}=0$ corresponds to $\varepsilon=0.057$; i.e., the predicted polarizations differ only slightly from the presented values for $a / M_{\mathrm{BH}}=0.5$, so for the source NGC 4258 instead of $6.6 \times 10^{3} \mathrm{G}$, corresponding to $a / M_{\mathrm{BH}}=0.5$ (see Table 1 ), we obtain $7.6 \times 10^{3} \mathrm{G}$ for limiting case $a / M_{\mathrm{BH}}=0$.

\section{The wavelength dependence of polarization of AGN and accretion disk models}

Polarization in AGNs can be intrinsic or extrinsic. Light scattering by a nonspherical distribution of electrons near the central engine of AGN is a basic intrinsic polarization mechanism; namely, an accretion disk is a typical example of a nonspherical distribution. Scattering by magnetically aligned dust grains in the interstellar medium of galaxies is the typical example of an extrinsic situation. The very important feature characterizing the polarized radiation from a magnetized accretion disk is the wavelength dependence of polarization degree that is very different from that of Thomson's scattering.

For a strong magnetic field strength, when $\delta_{\|, \perp} \gg 1$ the simple asymptotic formulas follow from Eqs. (9) and (10):

$$
\begin{aligned}
& p(\lambda) \approx \frac{p(0, \mu)}{\delta_{\|} \mu} \sim \frac{p(0, \mu)}{B_{\|} \lambda^{2}}, \\
& p(\lambda) \approx \frac{p(0, \mu)}{\delta_{\perp} \sqrt{1-\mu^{2}}} \sim \frac{p(0, \mu)}{B_{\perp} \lambda^{2}} .
\end{aligned}
$$

For turbulent atmospheres, with the existence of chaotic magnetic field component $B^{\prime}$, the corresponding asymptotic formulae follow from Eqs. (20). If the parameter of turbulence $C \gg \delta \gg 1$, then $p(\lambda) \sim 1 / \lambda^{4}$, if the level of magnetic fluctuations $\left\langle B^{\prime 2}\right\rangle$ is constant (see Sect. 3.2). Below we mainly consider the limiting spectra $\left(\delta_{\|, \perp} \gg 1\right)$ only for nonturbulent atmospheres. For turbulent atmospheres with a high value of $C$, the corresponding spectra are to be divided to $\lambda^{2}$.

The wavelength dependencies of the radiation flux and its polarization essentially stem from the radial distribution of the temperature in an accretion disk. For a standard accretion disk (Shakura \& Sunyaev 1973), radial dependence of the temperature takes the form: $T_{\mathrm{e}} \sim r^{-3 / 4}$. To get the integrated spectrum from the disk, we add up all of the Planck curves from each radius. If $T_{\mathrm{e}} \sim r^{-s}$ then the radiation flux (see Pringle \& Rees 1972; Shakura \& Syunyaev 1973; Gaskell 2008):

$F_{v} \sim v^{3-2 / s}$.

For a standard accretion disk ( $s=3 / 4)$, the flux (30) takes the known form $F_{v} \sim v^{1 / 3}$.

Substitution of $T_{\mathrm{e}} \sim r^{-s}$ into formula (13) leads to relation $R_{\lambda} \sim \lambda_{\mathrm{e}}^{1 / s}$ between the characteristic scale $R_{\lambda}$ and the effective wavelength of polarimetric observations. According to expression (11) we obtain $B_{\|, \perp}\left(R_{\lambda}\right) \sim \lambda^{-n / s}$. As a result, from Eqs. (31) and (11), we obtain the next wavelength dependence for the case of a strong Faraday depolarization:

$p(\lambda) \approx \frac{p(0, \mu)}{B_{\|, \perp} \lambda^{2}} \sim \lambda^{n / s-2}$.

As a result, the degree of observed polarization $p(\lambda)$ depends on both power-law indices $n$ and $s$. This comes from the depolarization effect of Faraday rotation of the polarization plane. In the absence of this effect, the polarization degree, independent of radiation flux $F_{\lambda}$, depends only on the inclination angle of the accretion disk. Of course, the usual Stokes parameters $I, Q$, and $U$ give the information on the radial distribution of the effective temperature (e.g. Eq. (32)).

For distribution of magnetic field in a standard accretion disk $(s=3 / 4)$ with $n=3 / 2\left(B_{\|, \perp} \sim R^{-3 / 2}\right)$, the polarization does not depend on the wavelength. For a standard accretion disk with $n=5 / 4$, mostly elaborated by Pariev et al. (2003), the wavelength dependence of the polarization is quite weak $p(\lambda) \sim \lambda^{-1 / 3}$. In this case the wavelength dependence of the Stokes polarized flux takes the form $p(v) F_{v} \sim v^{2 / 3}$. In the general case the formulae (32) and (33) give rise to relation

$p(v) F_{v} \sim v^{5-\frac{n+2}{s}}$.

The presented formulas allow us to test the various models of an accretion disk using the data of the wavelength dependence of polarization of AGN and quasars.

For the turbulent accretion disk with a high value of parameter $C$, formula (34) transforms to the expression

$p(v) F_{v} \sim v^{7-\frac{n+2}{s}}$.

The position angle values $\chi$, used in our formulae, denote the angles between the accretion disk plane and the observed direction of electric field wave oscillations. If it is impossible to estimate the disk inclination, we cannot obtain the theoretical $\chi$ from the observed position angle. The observed spectra of polarization degree $p_{\mathrm{obs}}(\lambda)$ and the differences of position angle $\chi_{\mathrm{obs}}\left(\lambda_{i}\right)-\chi_{\mathrm{obs}}\left(\lambda_{j}\right)$ are to coincide with the corresponding theoretical values $p(\lambda)$ and the differences in position angles $\chi\left(\lambda_{i}\right)-\chi\left(\lambda_{j}\right)$ for different wavelengths $\lambda_{i}$ and $\lambda_{j}$. These conditions make the estimation of magnetic field and unknown disk's inclination angle more precise than for the single wavelength observations.

\subsection{Discussion of some observational data}

Webb et al. (1993) and Impey et al. (1995) present the data of measurement $U B V R I$ polarizations of a sample of AGNs and QSOs. The position angle appears to be wavelengthindependent, suggesting that the polarization in a given object originates in a single physical process. In many cases the percentage of polarization increases with frequency. Authors have 
compared the polarized fluxes with the predictions of competing models of polarization in AGNs: synchrotron emission, scattering from electrons or different types of dust grains, and electron scattering in an accretion flow. In nine sources from this sample, the polarization seems to be the result of dust grain scattering. A number of these sources (NGC 4151, Mrk 509, NGC 5548, Mrk 290) has best characteristics of model due to electron scattering in an accretion disk or torus. It is interesting that, for these four sources, the slopes of $F_{v} \sim v^{\gamma}$ are close $(\gamma \simeq-1.37,-0.9$, $-0.86,-0.9$, respectively; see Webb et al. (1993). According to formula (32), we obtain the corresponding values of parameter $s=0.46,0.47,0.52,0.51$; i.e., they are near $s=0.5$.

The general shape of the polarization spectrum with parameter " $q$ " was determined by a least-squares fit proportional to $p(v) F_{v}=A v^{q}$. It appears that the slope of the wavelength dependence of polarized flux $q$ varies widely, between -2 and +1 , with a typical uncertainty of 0.3 .

What values of parameter $n$ corresponds to limit values of $q$, if we accept $s=1 / 2$ using formulas (34) and (35)? For nonturbulent accretion disk (Eq. (34)) we obtain

$n=\frac{1-q}{2}$.

This formula gives $n=1.5$ for $q=-2, n=1$ for $q=-1, n=0.5$ for $q=0$, and $n=0$ for $q=1$. Remember (see Pariev et al. 2003) that accretion disk with decreasing gas density at large distances $r$ corresponds to $n>1$. It seems the self-consistent models of Pariev et al. (2003) allow the values of $n \simeq 1-2$. The high values with $n>2$ correspond to a very sharp decrease the magnetic field inside the accretion disk. That is why we investigate the cases $1<n<2$ as physically acceptable according to the results of Pariev et al. (2003). From Eq. (32) we see that $p_{\lambda} \sim \lambda^{2(n-1)}$ at $s=1 / 2$. This means that the degree of polarization that diminishes with the increasing $\lambda$ cannot be explained inside the physical limitations, assumed above $(n>1$ corresponds to increasing polarization degree with $\lambda$ ).

For this reason we investigate what gives the very high magnetic turbulence. For this case, formula (32) transforms to expression $p(\lambda) \sim \lambda^{n / s-4}$. It leads to $p(\lambda) \sim \lambda^{2(n-2)}$ at $s=1 / 2$. As a result, our physically acceptable restriction $1<n<2$ allows us, in principle, to explain the decreasing degree of polarization. Taking the definition $p(v) F_{v} \sim v^{q}$ into account, we derive from Eq. (34) the following relation

$n=(7-q) s-2$.

For $s=0.5$ this expression gives $n=1$ for $q=1$, and $n=$ 1.5 for $q=0$. For $q=-2$ this formula gives $n=5 / 2$, which characterizes a very sharp decrease in magnetic field inside the accretion disk.

As an example, we now consider the continuum radiation of source NGC 4151. According to Webb et al. (1993), we have $\gamma \simeq-1.37$, and from Schmidt \& Miller (1980) the parameter $q$ is equal to $q \simeq-0.33$ (see Fig. 3 for the value $p(v) F_{v}$ in the interval $\lambda=(0.3-0.94) \mu \mathrm{m})$. From Eq. (31) we obtain $s=2 /(3-\gamma)$, which gives in this case $s \simeq 0.46$. Substitution of the values $q=-0.33$ and $s=0.46$ to Eq. (37) gives $n \simeq 1.37$. In nonturbulent case we have $n \simeq 0.45$, which corresponds to the increase in gas density with growing distance from the nucleus (see Pariev et al. 2003). In our case, when we know the observed spectra $F_{v} \sim v^{\gamma}$, and $p(v) F_{v} \sim v^{q}$, both Eqs. (34) and (35) give rise to the same expression $p(\lambda) \sim \lambda^{\gamma-q} \simeq \lambda^{-1.04}$. Thompson et al. (1979, see Fig. 3) present the polarization degree $p(\lambda) \%$ in the same interval $\lambda=(0.3-1) \mu \mathrm{m}$. The data have a rather wide distribution.
Using our dependence, we can approximate the polarization degree by the formula $p(\lambda) \simeq-0.5+0.7 \times \lambda^{-1.04}(\mu \mathrm{m})$, which fairly satisfactorily describes the mean value of the presented data.

\section{Conclusions}

We have presented the method for estimating the magnetic field strength at the event horizon of a supermassive black hole through the polarization of accreting disk emission. The polarized radiation arises from the scattering of emission light by electrons in a magnetized accretion disk. Due to Faraday rotation of the polarization plane, the resulting polarization degree differs essentially from the classical Thomson case, because the wavelength dependence of the polarization degree appears. This feature means that the magnetic field strength at the event horizon of a black hole can be estimated from polarimetric observations in the optical range.

For estimating observed polarization, we use the azimuthally averaged asymptotic expressions of the Stokes parameters for outgoing radiation, assuming that the accretion disk is optically thick and that the Milne problem takes place. Using these formulae, we discuss the wavelength dependence of the observed spectra of polarization degree. We also consider turbulent accretion disks when the magnetic field possess both regular component and chaotic one. Since the polarization spectrum of scattered radiation strongly depends on the accretion disk model, our results can be used to construct a realistic physical model of the AGN environment.

The estimates of the magnetic field strength of supermassive black holes in NGC 4258 are presented. We also found for the source NGC 4151 that the observed polarization degree spectrum can be satisfactorily explained by our mechanism with the high level of turbulent magnetic field. In this case the power-law regular magnetic field is $B \sim r^{-1.37}$ and $T_{\mathrm{e}} \sim r^{-0.46}$. In the cases where the observed polarization is the result of various mechanisms, such as light scattering in an accretion disk and a jet, our method can be considered as important part of the problem as a whole.

Acknowledgements. This research was supported by the RFBR (project No. 0702-00535a), the program of Prezidium of RAS "Origin and Evolution of Stars and Galaxies", the program of the Department of Physical Sciences of RAS "Extended Objects in the Universe" and by the Grant from President of the Russian Federation "The Basic Scientific Schools" NS-6110.2008.2. M.Yu. Piotrovich acknowledges the Council of Grants of the President of the Russian Federation for Young Scientists, grant No. 4101.2008.2.

We are very grateful to the anonymous referee for many remarks that allowed us to clarify the paper considerably.

\section{References}

Agol, E., \& Blaes, O. 1996, MNRAS, 282, 965

Barth, A. J., Tran, H. D., Brotherton, M. S., et al. 1999, AJ, 118, 1609

Blaes, O. 2003, in Accretion discs, jets and high energy phenomena in astrophysics, Les Houches Session LXXXIII, 2002, ed. V. Beskin et al. (N.Y.: Springer)

Blandford, R. D., \& Payne, D. C. 1982, MNRAS, 199, 883

Blandford, R. D., \& Znajek, R. L. 1977, MNRAS, 179, 433

Campbell, C. G. 2000, MNRAS, 317, 501

Cao, X. 1997, MNRAS, 291, 145

Chandrasekhar, S. 1950, Radiative transfer (Oxford: Clarendon press)

Chang, P., Quataert, E., \& Murray, N. 2007, ApJ, 662, 94

Connors, P. A., Piran, T., \& Stark, R. F. 1980, ApJ, 235, 224

Dolginov, A. Z., Gnedin, Yu. N., \& Silan'ev, N. A. 1995, Propagation and polarization of radiation in cosmic media (N.Y.: Gordon \& Breach)

Dovciak, M., Karas, V., \& Matt, G. 2004, MNRAS, 355, 1005 
Gaskell, C. M. 2008, Rev. Mex. Astron. Astrophys. (serie de Conferencias), 32, 1

Gnedin, Yu. N., \& Silant'ev, N. A. 1997, Basic Mechanisms of Light Polarization in Cosmic Media (Amsterdam: Hartwood)

Gnedin, Yu. N., Silant'ev, N. A., \& Shternin, P. S. 2006, Astron. Lett., 32, 39

Grandi, P., Malaguti, G., \& Fiocchi, M. 2006, ApJ, 642, 113 [arXiv: astro-ph/0511784]

Hawley, J. F., Gammie, C. F., \& Balbus, S. A. 1995, ApJ, 440, 742

Herrnstein, J. R., Moran, J. M., Greenhill, L. J., et al. 1997, ApJ, 475, L17

Herrnstein, J. R., Moran, J. M., Greenhill, L. J., et al. 1999, Nature, 400, 539

Herrnstein, J. R., Moran, J. M., Greenhill, L. J., \& Trotter, A. S. 2005, ApJ, 629, 719

Impey, C. D., Malkan, M. A., Webb, W., \& Petry, C. E. 1995, ApJ, 440, 80

Karas, V., Dovciak, M., \& Matt, G. 2004, 22th Texas Symposium on relativistiv astrophysics at Stanford University

Kochanek, C. S., Dai, X., Morgan, C., Morgan, N. \& Poindexter, S. 2006 [arXiv: astro-ph/0609112]

Königl, A. 1989, ApJ, 342, 208

Krolik, J. H. 2007 [arXiv:0709. 1489]

Kroll, N. A., \& Trivelpiece, A. W. 1973, Principles of plasma physics (N.Y.: McCraw-Hill)

Li, L. X. 2002, A\&A, 392, 469

Ma, R.-Y., Yang, F., \& Wang, D.-X. 2007, ApJ, 671, 1981

Martin, P. G. 1989, IAUS, 135, 55

Martin, P. G., Thompson, I. B., Maza, J., \& Angel, J. R. P. 1983, ApJ, 266, 470

Melia, F. 1992, ApJ, 387, L25

Modjaz, M., Moran, J. M., Kondratko, P. T., \& Greenhill, L. J. 2005, ApJ, 626, 104

Moran, J. 2008, ASPC, 395, 87

Morgan, C., Kochanek, C. S., Morgan, N. D. \& Falco, E. E. 2006, ApJ, 647, 874

Morgan, C. W., Kochanek, C. S., Dai, X., Morgan, N. D., \& Falco, E. E. 2008, ApJ, 689, 755

Novikov, I. D., \& Thorne, K. 1973, Black Holes, ed. C. De Witt-Morette, \& B. C. De Witt (New York: Gordon and Breach)
Ogilvie, G. I., \& Livio, M. 2001, ApJ, 553, 158

Pariev, V. I., Blackman, E. G., \& Boldyrev, S. A. 2003, A\&A, 407, 403

Pariev, V. I., \& Colgate, S. A. 2007, ApJ, 658, 129

Poindexter, S., Morgan, N., \& Kochanek, C. 2008, ApJ, 673, 34

Pringle, J. E., \& Rees, M. J. 1972, A\&A, 21, 1

Pringle, J. E., Antonucci, R. R. J., Clarke, C. J., et al. 1999, ApJ, 526, L9

Reynolds, S. R., Nowak, M. A., Markoff, S., et al. 2008 [arXiv: 0810. 2543]

Rohlfs, K., \& Wilson, T. L. 1996, Tools of Radio Astronomy (Berlin: Springer)

Satyapal, S., Dudik, R. P., O’Halloran, B., \& Gliozzi, M. 2005, ApJ, 633, 865

Sano, T., Inutsiko, S.-I., Turner, N. J., \& Stone, J. M. 2004, ApJ, 605, 321

Schmidt, G. D., \& Miller, J. S. 1980, ApJ, 240, 759

Serkowskii, K. 1973, IAUS, 52, 145

Shakura, N. I., \& Sunyaev, R. A. 1973, A\&A, 24, 337

Shapiro, S. 2007 [arXiv: 0711. 1537]

Shternin, P. S., Gnedin, Yu. N., \& Silant'ev, N. A. 2003, Astrofizika, 46, 433

Silant'ev, N. A. 1994, JQSRT, 52, 207

Silant'ev, N. A. 2002, A\&A, 383, 326

Silant'ev, N. A. 2005, A\&A, 433, 1117

Silant'ev, N. A. 2007, Astron. Rep., 51, 67

Smith, J. E., Young, S., Robinson, A., et al. 2002, MNRAS, 355, 773

Thompson, I., Landstreet, J. D., Angel, J. R. P., et al. 1979, ApJ, 229, 909

Turner, N. J., Stone, J. M., Krolik, J. H., \& Sano, T. 2003, ApJ, 593, 992

van Kampen, N. G. 1981, Stochastic processes in Physics and Chemistry (Amsterdam: North Holland)

Webb, W., Malkan, M., Schmidt, G., \& Impey, C. 1993, ApJ, 419, 494

Wang, D.-X., Xiao, K. \& Lei, W.-H. 2002, MNRAS, 335, 655

Wang, D.-X., Ma, R.-Y., Lei, W.-H., \& Yao, G.-Z. 2003,

ApJ, 595, 109

Wilkes, B. J., Schmidt, G. D., Smith, P. S., et al. 1995,

ApJ, 455, L13

Zhang, D., \& Dai, Z. G. 2008, MNRAS, 388, 1409

Zhang, W. M., Lu, Y., \& Zhang, S. N. 2005, Chin. J. Astron. Astrophys. , 5, Suppl., 347 